\title{
Comprehensive mapping of the cell response to $E$. coli infection in porcine intestinal epithelial cells pretreated with exopolysaccharide derived from Lactobacillus reuteri
}

\author{
L’udmila Tkáčiková ${ }^{1}$, Evelína Mochnáčová ${ }^{2}$, Punit Tyagi ${ }^{2}$, Zuzana Kiššová ${ }^{1}$ and Mangesh Bhide ${ }^{2,3^{*}}$ (1)
}

\begin{abstract}
Bacterial exopolysaccharides (EPSS) are known to modulate immunity. To date, a plethora of studies have reported the effect of EPSs on intestinal cells; however few works have revealed a complete picture of the signalling events in intestinal epithelial cells induced by bacterial EPS. Here, using transcriptomics, we comprehensively mapped the biological processes in porcine intestinal epithelial cells challenged with EPS derived from Lactobacillus reuteri alone, enterotoxigenic Escherichia coli (ETEC) or ETEC after pretreatment with EPS. The Gene Ontology analysis of differentially expressed genes (DEGs) showed that ETEC is able to evoke biological processes specifically involved in cell junction reorganization, extracellular matrix degradation, and activation of the innate immune response through the activation of pattern recognition receptors, such as TLRs and CTRs. A total of 495 DEGs were induced in ETEC-challenged cells. On the other hand, EPS pretreatment was able to attenuate overexpression of the genes induced by ETEC infection. The most relevant finding of this study is that EPS has a suppressive effect on the inflammatory response evoked by ETEC infection. On the basis of high-throughput RNA-seq, this report is the first to describe the effects of EPSs derived from $L$. reuteri used as a pretreatment of global gene expression in porcine epithelial cells.
\end{abstract}

\section{Introduction}

Bacterial exopolysaccharides (EPSs) are extracellular polysaccharides that play pivotal roles in the protection of bacteria and adhesion to host cells. EPSs are either covalently attached as a capsule to the surface of bacteria or released into the environment [1]. Among the beneficial bacteria, Lactobacillus represents one of the best producers of EPS. Exopolysaccharides produced by lactobacilli have not only positive effects on their producers $[2,3]$

\footnotetext{
*Correspondence: bhidemangesh@gmail.com

${ }^{2}$ Laboratory of Biomedical Microbiology and Immunology, University of Veterinary Medicine and Pharmacy in Kosice, Kosice, Slovakia

Full list of author information is available at the end of the article
}

but also immunomodulatory effects on the gut mucosal immune system [4-6]. Exopolysaccharides stimulate the immune response in intestinal epithelial cells (IECs) through the activation of $\mathrm{C}$-type lectin receptors (CLRs). The activation of IECs results in the induction of a broad range of cytokines and chemokines, including interleukins, TNF, growth factors and beta-defensins [7]. Thus, IECs play important roles in the activation of dendritic cells that control innate and acquired immune responses [8].

Enterotoxigenic Escherichia coli (ETEC) is one of the most common causes of post-weaning diarrhea in pigs [9, 10]. ETEC interacts with epithelial cells, colonizes the small intestine and secretes thermostable (ST) or 
thermolabile (LT) enterotoxins, inducing acute intestinal diarrhea and inflammation [11]. In addition, ETEC triggers inflammatory responses mediated through other pathogen-associated molecular patterns, such as lipopolysaccharides (LPSs), that significantly contribute to intestinal tissue injury during infection $[9,11]$. ETEC infection is responsible for economic losses in the pig industry mainly due to high mortality, morbidity, growth retardation and treatment costs [12]. Thus, it is necessary to protect piglets against ETEC infection by modulating their gut immunity with prebiotics, probiotics or the products of beneficial bacteria such as EPSs.

We have previously observed that the EPS isolated from Lactobacillus reuteri strain L26 Biocenol possesses cytokine-modulating activity. This EPS is a homopolymer consisting of $\alpha$-D-glucose $(1 \rightarrow 3)$ and $(1 \rightarrow 6)$ glycosidic bonds at a ratio of $1.3: 1$ with a molecular weight $(\mathrm{Mw})$ of $8.2 \times 10^{5} \mathrm{Da}$ [6]. To date, scarce literature is available that presents a complete picture of the cell response (at the transcriptomic level) to the EPS derived from Lactobacillus. The plausible protective effect of EPS mediation of the immune response against $E$. coli infection has been documented previously, however, with a small-scale experimental approach [6].

To date, few studies have addressed the cell response to lactobacilli, E. coli or bacterial toxins at the transcriptomic level. Kobayashi et al. [13] used a microarray to investigate the response of porcine intestinal epithelial (PIE) cells to the ST toxins of ETEC and Lactobacillus jensenii. The transcriptomic response of PIE cells to the viral molecular associated pattern polyinosinic-polycytidylic acid and immunobiotic strains Lactobacillus rhamnosus and Lactobacillus plantarum was previously studied [14]. Similarly, differences in genome-wide gene expression induced by a mixture of three Lactobacillus strains (L. rhamnosus, L. plantarum, and Lactobacillus paracasei) in intestinal porcine epithelial cells (i.e., IPEC-1 cells) were also reported [15]. In another study, gene expression in IPEC-J2 cells exposed to microalgal extracts with or without challenge with ETEC was reported [16]. To our knowledge, no work has been published thus far that maps a complete picture of the IPEC-1 cell response at the transcriptomic level to E. coli challenge with or without pretreatment of EPS isolated from the probiotic lactobacilli.

Therefore, the objective of this study was to map a comprehensive picture of the gene expression in IPEC-1 cells challenged with ETEC with or without pretreatment with EPS. We hypothesized that transcriptomic analyses using IPEC-1 cells could provide valuable data to understand the mechanisms involved in the protective effect of EPS mainly against intestinal inflammatory damage caused by E. coli.

\section{Materials and methods Bacterial strains}

Lactobacillus reuteri L26 Biocenol (CCM 8616) was provided by Radomíra Nemcová, Institute of Microbiology and Gnotobiology, University of Veterinary Medicine and Pharmacy in Košice, Slovakia. L26 was cultured in modified de Man-Rogosa-Sharpe medium (MRS; HiMedia, India) containing 10\% sucrose (Mikrochem, Slovakia). For EPS extraction, the modified MRS medium was inoculated with overnight culture of $L$. reuteri $(10 \% \mathrm{v} / \mathrm{v})$ and incubated for $48 \mathrm{~h}$ at $37{ }^{\circ} \mathrm{C}$.

Hemolytic ETEC strain $11501\left(\mathrm{O} 149: \mathrm{K}^{+} 8^{+} \mathrm{STb}^{+}\right.$, $\mathrm{LT}^{+}$, provided by Martin Faldyna, Veterinary Research Institute, Brno, Czech Republic) was cultivated overnight in lysogeny broth (LB; Sigma-Aldrich, USA) at $37{ }^{\circ} \mathrm{C}$ with constant shaking $(100 \mathrm{rpm})$. The LB was inoculated with $1 \%(\mathrm{v} / \mathrm{v})$ of a $16-\mathrm{h}$ ETEC culture and incubated for the next $3 \mathrm{~h}$ at $37^{\circ} \mathrm{C}$ with constant shaking. The number of $E$. coli was determined using a spectrophotometer at $\mathrm{OD}_{600}$ based on the following equation: $\mathrm{OD}_{600}$ of $1.0=8 \times 10^{8}$ cells $/ \mathrm{mL}$.

\section{Extraction and purification of the EPSs}

The EPS was isolated and purified from $L$. reuteri L26 exactly as described in our previous report [6].

\section{IPEC-1 cell culture and experimental design}

The IPEC-1 cell line was grown in 6-well culture plates in medium containing Dulbecco's modified Eagle medium/F-12 (Sigma-Aldrich) supplemented with 5\% foetal bovine serum (FBS; Lonza, Switzerland), $5 \mathrm{ng} /$ $\mathrm{mL}$ epidermal growth factor (BD Biosciences, USA), $10 \mu \mathrm{g} / \mathrm{mL}$ insulin, $10 \mu \mathrm{g} / \mathrm{mL}$ transferrin, and $10 \mathrm{ng} / \mathrm{mL}$ selenium (Lonza) at $37{ }^{\circ} \mathrm{C}$ in a fully humidified atmosphere with $5 \% \mathrm{CO}_{2}$. Upon reaching $70 \%$ confluency, the cells were washed with sterile phosphate-buffered saline, and EPS $(0.1 \mathrm{mg} / \mathrm{mL}, 1 \mathrm{~mL}$ each well) reconstituted in IPEC-1 cell medium without FBS was added. After $4 \mathrm{~h}$ of preincubation with the EPS, the cells were challenged with ETEC (multiplicity of infection: 50:1) without refreshing of the medium for $45 \mathrm{~min}$. Subsequently, the monolayers were washed with sterile PBS and stored at $-20^{\circ} \mathrm{C}$ until further use.

\section{RNA isolation and integrity}

mRNA from the IPEC-1 cells was isolated using an RNeasy Mini kit (Qiagen, Germany) according to the manufacturer's instructions. DNaseI (Qiagen) treatment was incorporated during RNA isolation. The integrity of the RNA was monitored using capillary 
electrophoresis (Fragment Analyzer, Advanced Analytical Technologies, Inc., USA).

\section{Preparation of the library}

A total of 250 ng of RNA was reverse transcribed with oligo-dT primers for the synthesis of first-strand cDNA using a QuantSeq 3' mRNA-seq library prep kit (Lexogen, Austria). All the steps described below were completed exactly following the manufacturer's instructions. The RNA template was removed with RNA removal solution (RS buffer, Lexogen), and the second strand was synthesized using a random hexamer primer that contains Illumina-compatible linker sequences at its $5^{\prime}$ end. The double-stranded DNA libraries were purified using magnetic beads provided in the kit. Each library was amplified by PCR using unique, single-indexing i7 primers to add the complete adapter sequence required for cluster generation and to generate sufficient DNA for sequencing and quality control. The number of PCR cycles for each library was determined using a PCR Add-on Kit for Illumina (Lexogen). The cycles used for library amplification were as follows: IPEC-1 cells challenged with ETEC-20 cycles, IPEC-1 cells treated with EPS -20 cycles, IPEC-1 cells pretreated with EPS and challenged with ETEC20 cycles, and non-treated cells-17 cycles. Amplified libraries were purified using the magnetic beads supplied in the kit. The quality of the libraries and length of the fragments were determined on a fragment analyser.

\section{NGS sequencing}

Libraries were sequenced on a Illumina NextSeq, single-end, $75 \mathrm{bp}$, to a minimal depth of 8 million reads per sample. FASTQ files were processed and aligned to the reference genome using STAR alignment software [17]. The preprocessing includes adaptor trimming and removal of the initial 10 bases (recommended for QuantSeq as these bases are random priming sites). Reads were counted with STAR V 2.5.2b. To perform differential gene expression analysis, edgeR, the open source $R$ package, version 3.12 was used [18]. Low read counts, with less than 3 CPM (count per million), were filtered out using the filterByExp function of the edgeR package. The identification of differentially expressed genes (DEGs) was accomplished by using the glmTreat and glmQLFit (quasi-likelihood, QL) functions of edgeR in the $\mathrm{R}$ package, considering log fold change (logFC) values greater than \pm 1.2 and FDR values less than 0.05 .

\section{Quantitative expression analysis by RT-PCR}

RNA was reverse transcribed into cDNA using random hexamers (Thermo Fisher Scientific). Briefly, $1 \mu \mathrm{g}$ of RNA and 100 pMol of random hexamers were mixed and incubated for $5 \mathrm{~min}$ at $65^{\circ} \mathrm{C}$. Subsequently, $4 \mu \mathrm{L}$ of $5 \times$ reaction buffer, $2 \mu \mathrm{L}$ of dNTP $(10 \mathrm{mM}), 1 \mu \mathrm{L}$ of RevertAid reverse transcriptase (200 U) (Thermo Fisher Scientific, USA) and $0.5 \mu \mathrm{L}$ RiboLock RNase inhibitor (20 U) (Thermo Fisher Scientific) were added. The reaction mixture was incubated for $10 \mathrm{~min}$ at $25^{\circ} \mathrm{C}, 1 \mathrm{~h}$ at $42{ }^{\circ} \mathrm{C}$, and 10 min at $70{ }^{\circ} \mathrm{C}$.

Primers used in qRT-PCR were designed using Geneious Pro software (Biomatters, USA); they are presented in Table 1. The reaction mix of qRT-PCR consisted of $6 \mathrm{ng}$ of cDNA, $1 \times$ iQ SYBR Green Supermix (Bio-Rad, Hercules, CA), gene-specific primers (12.5 pMol each) and RNase-free water to a total volume of $20 \mu \mathrm{L}$. Each reaction was performed in triplicate. The amplification cycles were as follows: $95^{\circ} \mathrm{C}-10 \mathrm{~min}, 35 \times\left[95^{\circ} \mathrm{C}-30 \mathrm{~s}\right.$., $55-60{ }^{\circ} \mathrm{C}-30 \mathrm{~s}$ (annealing temperature varied according to the primers used), $72{ }^{\circ} \mathrm{C}$ for $30 \mathrm{~s}$ (signal capture)], melting curve from 60 to $95{ }^{\circ} \mathrm{C}-0.3 \%$ temperature increment/s. (StepOnePlus, Thermo Fisher Scientific, USA). The gene expression $(\Delta \Delta \mathrm{Ct})$ was normalized to that of the housekeeping gene, $\beta$-2-microglobulin (B2M). The $\Delta \Delta \mathrm{Ct}$ values were converted to $\log \mathrm{FC}$ values using an online server [19]. The expression values for the DEGs obtained from RNA-seq and qRT-PCR were correlated with Pearson correlation coefficients (PCCs) using an online server [20].

Table 1 PCR primers used in this study

\begin{tabular}{|c|c|c|c|c|c|}
\hline Genes & Forward primer $\left(5^{\prime} \rightarrow 3^{\prime}\right)$ & Reverse primer $\left(5^{\prime} \rightarrow 3^{\prime}\right)$ & $\mathrm{T}_{\mathrm{m}}\left({ }^{\circ} \mathrm{C}\right)$ & $\begin{array}{l}\text { Product } \\
\text { length (bp) }\end{array}$ & $\begin{array}{l}\text { Primer designed using } \\
\text { sequence from NCBI repository } \\
\text { GenelD }\end{array}$ \\
\hline B2M & CCGCATCTCCGTGTACTACAA & CAGCCCCTTCTGTATAGTGGC & $55 / 60$ & 197 & 100153507 \\
\hline AHCYL & TTGACCCCATCTGTGCTCTG & TGGCCCATATTGCACACGAT & 55 & 172 & 100512899 \\
\hline NFKB1 & TCGCTGCCAAAGAAGGACAT & TAGCGTTCAGACCTTCACCG & 55 & 102 & 751869 \\
\hline TRPC 1 & GCCTCCGACATTCCAGGTTT & TACATTGCCGGGCTAGTTCC & 55 & 180 & 100156938 \\
\hline YWHAZ & CCCAGAGAAAGCCTGCTCTC & TTCСССТССTTCTCCTGCTT & 55 & 181 & 780440 \\
\hline PSME1 & AGTATTTCTCTGAGCGGGGC & ATCCCGGTACTCTGCCTCAT & 55 & 107 & 397572 \\
\hline EHMT2 & AAGTGCAGCATTTCCGCATG & GAACCCAACTCCTCCGACAG & 60 & 115 & 100124382 \\
\hline
\end{tabular}




\section{Data analysis}

The logical relation of DEGs in the group with a negative control background was calculated using Excel (MS office), and a Venn diagram was constructed. To group DEGs into GO biological processes, the Reactome server was used [21], and to construct heat maps, the Heatmapper server was used [22]. Signalling pathways were downloaded from the KEGG server [23], and the DEGs involved in the KEGG pathways were manually highlighted.

\section{Results}

\section{Differentially expressed genes and validation}

In total, 495 genes were differentially expressed in the IPEC-1 cells challenged with ETEC, among which 348 (70.3\%) genes were upregulated and 147 (29.7\%) were downregulated (Figure 1, Additional file 8: Data set 1.1). A total of 119 genes were found to be differentially expressed in EPS-treated cells (98 genes upregulated and 21 genes downregulated) (Figure 1, Additional file 8: Data set 1.2). In the IPEC-1 cells treated with EPS and then challenged with ETEC, 130 (81.8\%) genes were upregulated, and 29 (18.2\%) genes were downregulated (Table 2, Figure 1 and Additional file 8: Data set 1.3). It is important to note that 336 genes were differentially expressed in the E. coli-infected cells that were not found to be differentially expressed in the cells pretreated and then challenged with $E$. coli. It is also noteworthy that 3 genes (Wnt inhibitory factor 1, Zinc finger $\mathrm{CCCH}$-type antiviral protein 1 isoform 1 and Zinc finger protein 181 isoform X2) were uniquely expressed among the cells treated only with EPS (Figure 1, Additional file 8: Data set 1.2). The common DEGs found among the cells challenged with different treatments are listed in Additional file 8: Data sets 1.4 to 1.6. When searching for common DEGS in all the cells subjected to one of the three

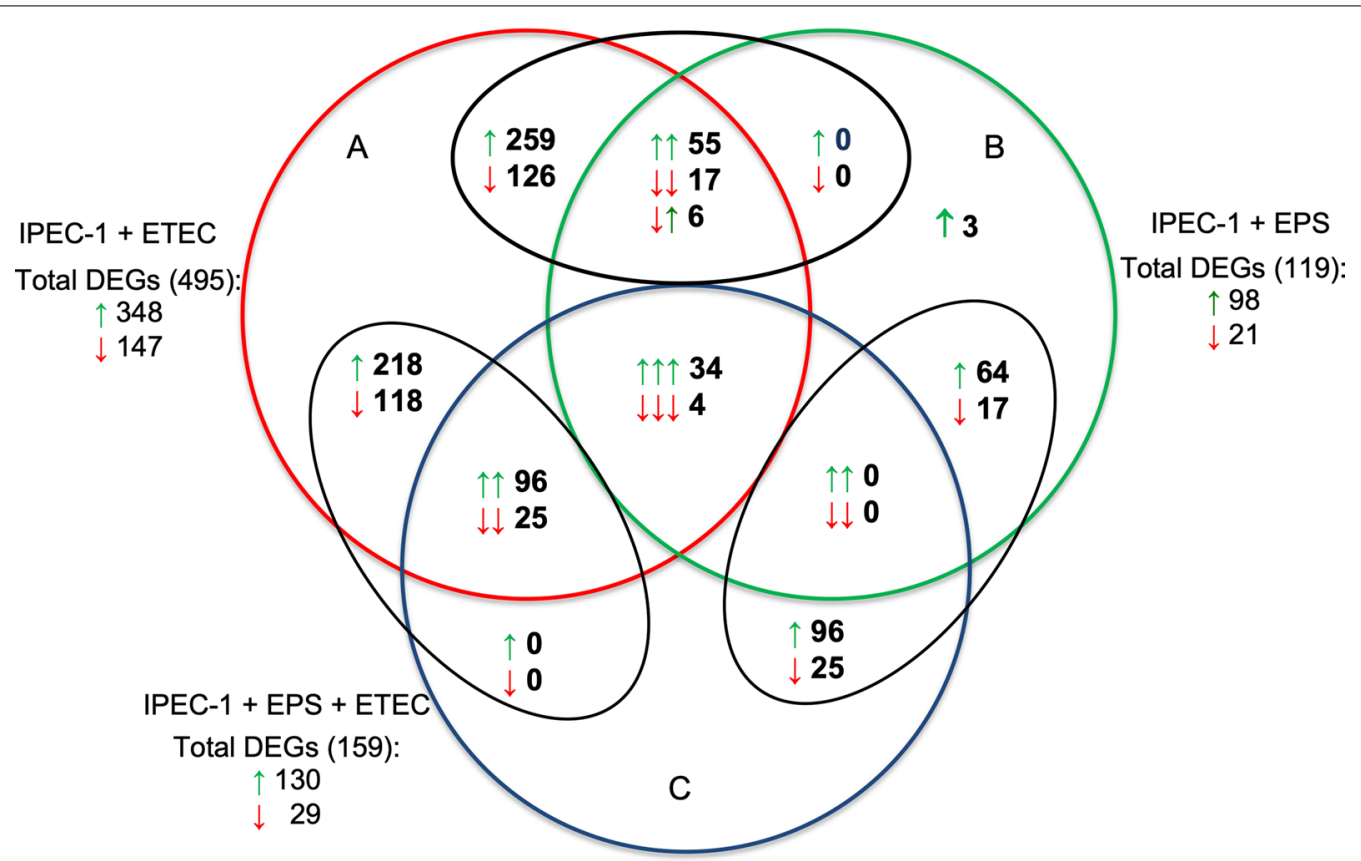

Figure 1 Venn diagram presenting the number of DEGs in the IPEC-1 cells challenged with ETEC (A), treated with EPS (B), or pretreated with EPS before ETEC challenge (C). Green arrows—upregulated DEGs. Red arrows—downregulated DEGs.

Table 2 Number of DEGs found in the cells induced with different treatments

\begin{tabular}{|c|c|c|c|c|c|}
\hline & \multirow[t]{2}{*}{ Total found DEGs } & \multicolumn{2}{|l|}{ Upregulated } & \multicolumn{2}{|l|}{ Downregulated } \\
\hline & & Number of DEGs & $\%$ & Number of DEGs & $\%$ \\
\hline IPEC -1 cells challenged with ETEC & 495 & 348 & 70.3 & 147 & 29.7 \\
\hline IPEC-1 cells treated with EPS & 119 & 98 & 82.4 & 21 & 17.6 \\
\hline $\begin{array}{l}\text { IPEC-1 cells pretreated with EPS and then chal- } \\
\text { lenged with ETEC }\end{array}$ & 159 & 130 & 81.8 & 29 & 18.2 \\
\hline
\end{tabular}


treatments, we found only 38 DEGs (34 upregulated and 4 downregulated genes) (Figure 1, Additional file 8: Data set 1.7).

To validate the results obtained from RNA-seq, the differential expression of 6 representative genes was analysed with qRT-PCR. The results obtained from both techniques were consistent (Figure 2) as determined ompared with Pearson correlation coefficient (PCC) $(r=0.712$ for IPEC- 1 cells exposed to ETEC; $r=0.728$ for IPEC- 1 cells exposed to EPS; and $r=0.455$ for IPEC- 1 cells exposed to EPS and ETEC; $p<0.01$ ). Following validation of the results, DEGs were segregated according to the GO biological process category using a peer-reviewed server-Reactome [21].

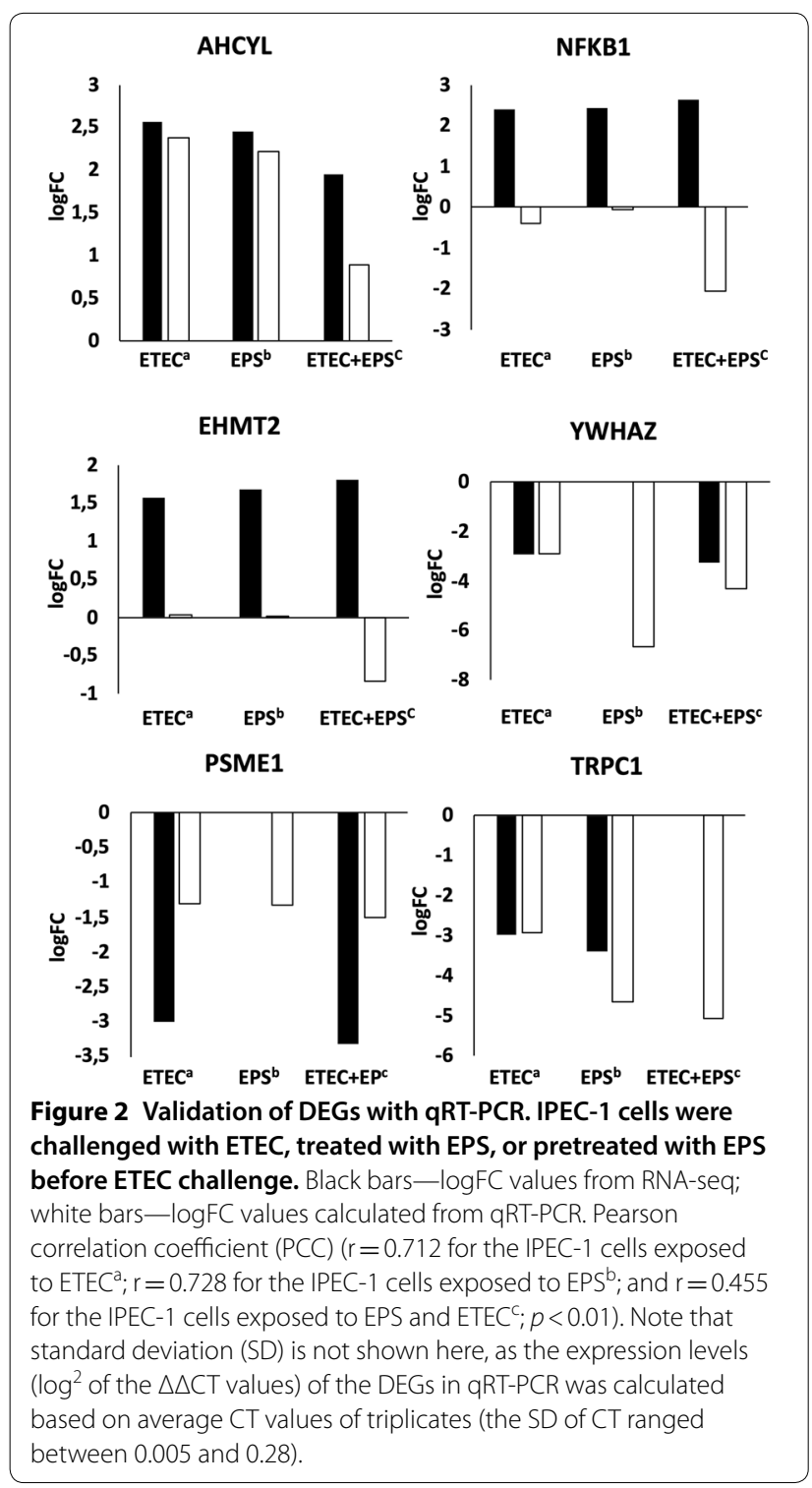

The results from segregating the DEGs according to GO biological process category, performed with the Reactome server, are presented in Additional file 1. Treatment with E. coli or EPS caused the induction of genes involved in several pathways (pathway identifiers are listed in Additional file 8: Data sets 1.8 to 1.10); however, here, we present the pathways that were directly related to the pathogenies of ETEC. These pathways are involved in cell junction organization, extracellular matrix (ECM) organization, the innate immune response, the CLRrelated pathway, TLR cascades and cytokine signalling.

\section{DEGs involved in cell junction organization}

The intestinal epithelial barrier plays an essential role in host defence against infections. The epithelial cell-cell junctional system comprises adherent junctions (AJs), tight junctions (TJs) and desmosomes [24], the disruption of which leads to an increase in the permeability of the barrier. Enterotoxins produced by ETEC strains may cause morphological changes in the intestinal mucosa, including the reorganization of cellular junctional proteins; thus, DEGs involved in this biological process are of main importance for this study. Six genes (CLDN20, PARD6B, CD151, CDH11, CLDN2 and F11R) related to the GO biological process "Cell junction organization" were upregulated by E. coli infection (Figure 3), whereas

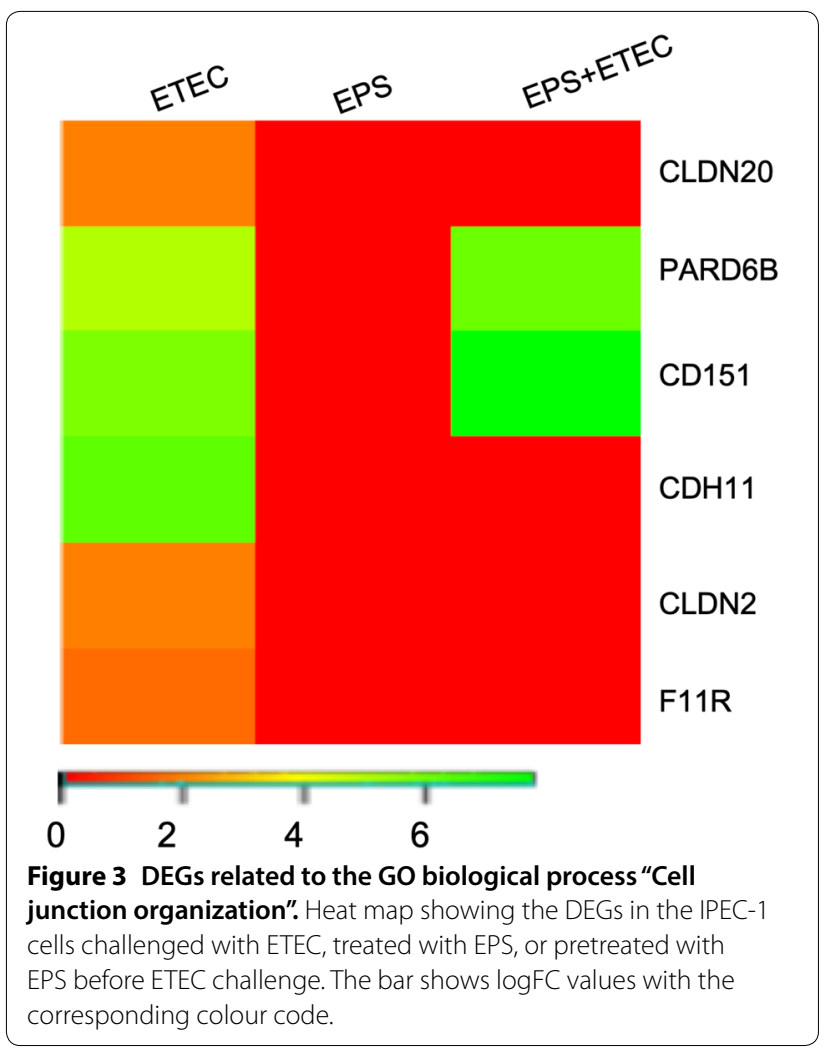


these genes were not induced by EPS (Additional file 2). A significant upregulation of CDH11 ( $\operatorname{logFC}$ of 6.33), encoding cell adhesion protein cadherin 11, observed in the cells infected with $E$. coli was abolished by EPS pretreatment before ETEC challenge. Cadherin 11 participates in the formation of AJs as is CLDN2 and CLDN20 (both $\log$ FC of 1.95). EPS pretreatment also caused the upregulation of F11R (encoding junctional adhesion molecule A, JAM-A) only in the infected cells without EPS pretreatment (logFC of 1.66). JAM-A is an important molecule in the formation of epithelial TJs [25].

Notably, EPS pretreatment before ETEC challenge induced the expression of CD151 ( $\operatorname{logFC}$ of 7.76) and PARD6B ( $\operatorname{logFC}$ of 6.14) genes. CD151 (a tetraspanin) participates in the formation of hemidesmosomes, the specialized multiprotein junctional complex that connects the keratin cytoskeleton of epithelial cells to the extracellular matrix and plays a critical role in the maintenance of tissue structure [26], while PARD6B (Par-6 family cell polarity regulator beta) participates in the formation of TJs [27].

\section{DEGs involved in extracellular matrix (ECM) organization}

ETEC enterotoxins cause morphological changes in the intestinal mucosa; thus, the regulation of the genes related to the GO biological process "Extracellular matrix organization" were analysed (Figure 4). Seventeen genes were induced in this GO process. E. coli infection resulted in the upregulation of the genes encoding proteases, which participate in the degradation of ECM, namely, ADAM10 (logFC of 2.62), ADAM17 (logFC of 8.75), MMP9 and MMP14 (both with a $\operatorname{logFC}$ of 2.58), CAPN7 and CAST (both with logFC of 2.20). The expression of these proteases remained unchanged after EPS pretreatment prior to E. coli infection, except MMP14 ( $\log \mathrm{FC}$ of 2.47). This finding indicates that the EPS pretreatment reduced the tissue damage caused by the ETEC infection. Although the genes involved in the degradation of ECM were upregulated, the expression of the ELN and COL1A2 genes encoding structural components of the ECM was changed only in the cells challenged with ETEC (Figure 4, Additional file 3). The ELN gene, encoding elastin, which contributes to the structural integrity of the ECM, was downregulated (logFC of -3.29 ), and the collagen type I alpha 2 chain-encoding gene, COL1A2, was upregulated (logFC of 1.90). Two small leucine-rich proteoglycans (SLRPs), decorin and asporin, encoded by the DCN and ASPN genes, respectively, were induced in the EPS-treated cells. SLRPs interact with different cell surface receptors, cytokines, growth factors and other ECM components, leading to the modulation of cellular functions. Decorin interacts with collagen and acts as a sink for all three isoforms of TGF-beta [28]. Degradation

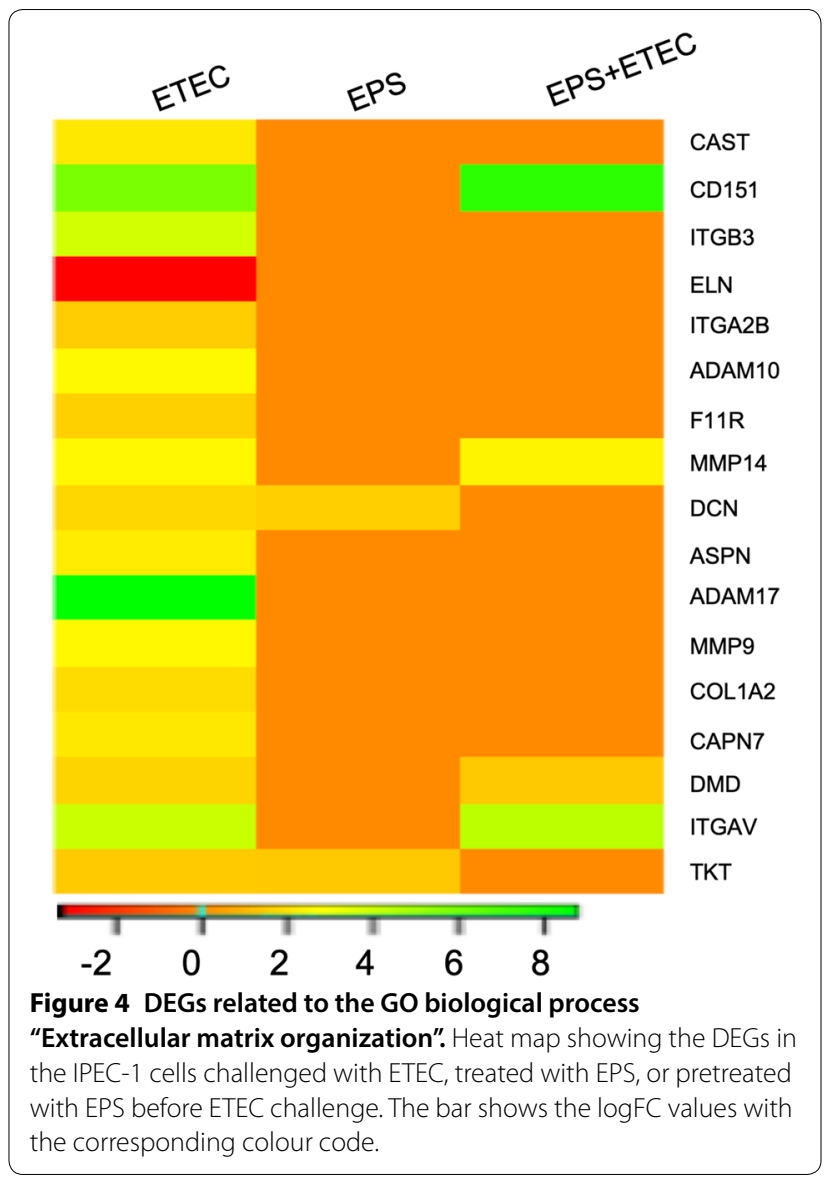

of decorin by matrix metalloproteinases results in the release of TGF-beta [29]. DCN was upregulated in the E. coli-infected cells (logFC of 1.81) and EPS-treated cells ( $\log$ FC of 1.59), whereas ASPN was upregulated only in the cells challenged with ETEC (logFC of 2.30) (Figure 4). The expression of three integrins (ITGAV, ITGB3 and ITGA2B) was altered in the E. coli-infected cells (Figure 4). Integrins are heterodimeric transmembrane receptors that mediate cell adhesion and bind extracellular matrix glycoproteins such as laminins and collagens in basement membranes or connective tissue components such as fibronectin [30]. Two of these genes, ITGB3 (logFC of 3.77) and ITGA2B ( $\operatorname{logFC}$ of 1.58$)$, were upregulated in the cells challenged with ETEC, while this upregulation was abolished by pretreating cells with EPS before infection (Figure 4). Such attenuation was not observed in the case of the ITGAV gene in the pretreated cells (ETEC-logFC of 3.99 after pretreatment and ETEC- $\log F C$ of 4.35).

\section{DEGs related to innate immune response}

In total, 68 genes (21 downregulated and 47 upregulated) categorized in the GO biological process "Innate immune 
system" were induced by ETEC challenge (Figure 5). On the other hand, ETEC challenge induced only 22 genes (6 downregulated and 16 upregulated) in the EPS-pretreated cells. In the cells treated only with EPS, 14 genes (3 downregulated and 11 upregulated) were found with altered expression. In the E. coli-infected cells, the highest fold-change was observed for ATOX1 ( $\operatorname{logFC}$ of 7.55), encoding antioxidant 1 copper chaperone, which binds and delivers cytosolic copper to the copper-ATPase proteins [31]. The SOCS1 gene, encoding suppressor of cytokine signaling 1 , was the most downregulated (logFC of -4.38); SOCS1 is involved in the negative regulation of cytokines that signal through the JAK/STAT3 pathway. Expression of the ATOX1 and SOCS1 genes was abolished by the EPS treatment and ETEC challenge of in the pretreated cells (Figure 5).

In the cells pretreated with EPS and then infected with $E$. coli, the highest fold change was observed for the gene encoding CD14 ( $\log \mathrm{FC}$ of 7.87 ), while the gene encoding CD180 was the downregulated to the greatest extent ( $\operatorname{logFC}$ of -4.26$)$. The CD14 molecule is a coreceptor for bacterial LPS [32], and CD180 (also known as RP105) is a negative regulator of TLR4 [5]. The expression of these genes was also altered in the E. coli-infected cells (CD14, $\operatorname{logFC}$ of 5.95; CD180, $\operatorname{logFC}$ of -3.73) but not in the cells treated only with EPS. Notably, treatment of the cells only with EPS induced TLR4 (logFC of 2.77). The expression of this gene was also upregulated in ETEC-challenged cells (both without pretreatment, $\operatorname{logFC}$ of 2.06, or with pretreatment, $\operatorname{logFC}$ of 2.62). The gene encoding CHUK, a component in the inhibitor of the nuclear factor kappa $\mathrm{B}$ kinase complex, was the downregulated to the greatest extent ( $\operatorname{logFC}$ of -3.85$)$. The expression of the CHUK gene was also downregulated in the ETEC-challenged cells (logFC of -3.47 ) but not in the cells pretreated with EPS and infected with ETEC (Figure 5).

\section{DEGs related to C-type lectin receptors}

C-type lectin receptors (CLRs) constitute a large superfamily of proteins that act as pattern-recognition receptors for pathogen-derived carbohydrates. Twelve DEGs (6 downregulated and 6 upregulated) related to the GO

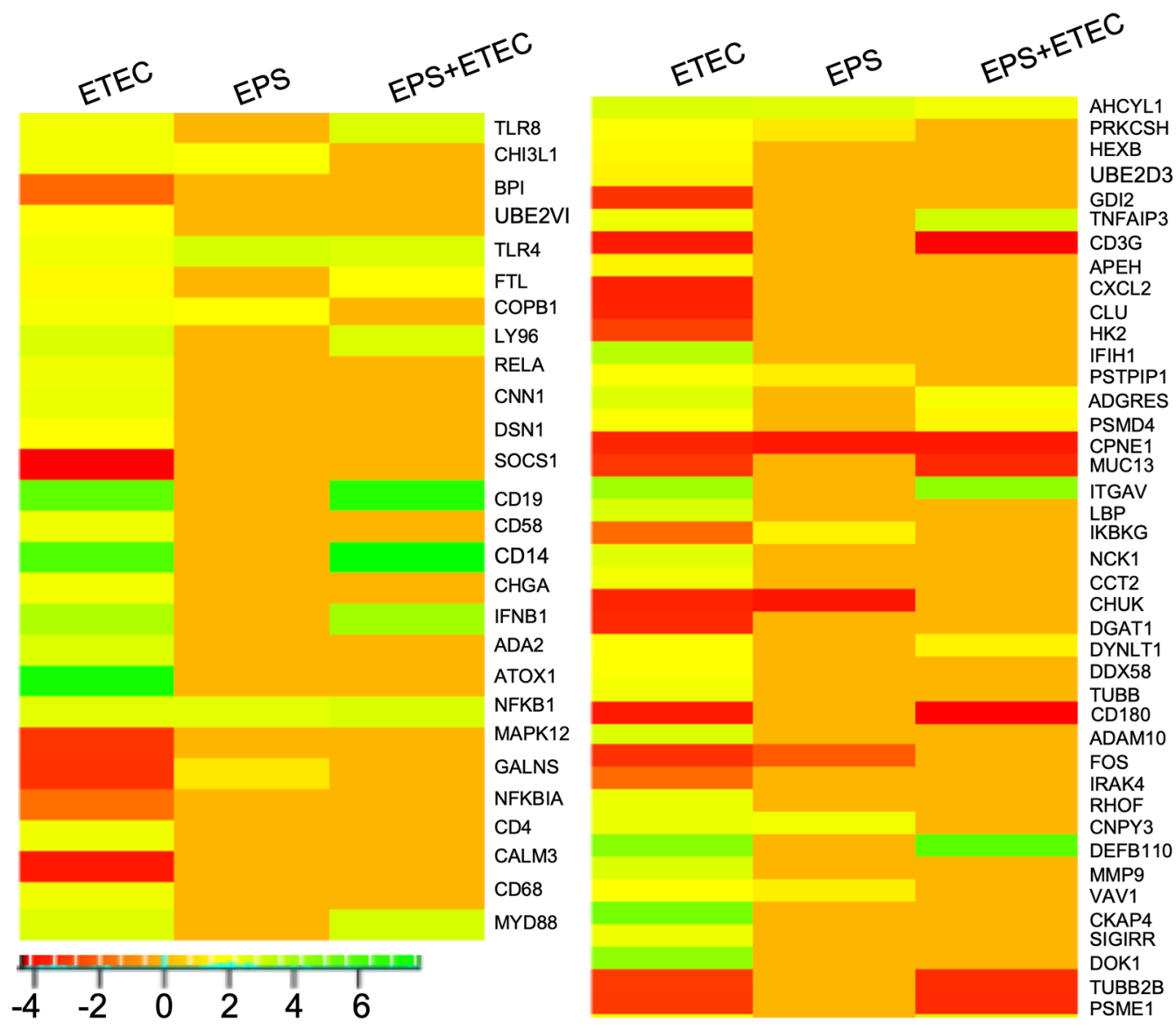

Figure 5 DEGs related to the GO biological process "Innate immune system". Heat map showing the DEGs in the IPEC-1 cells challenged with ETEC, treated with EPS, or pretreated with EPS before ETEC challenge. The bar shows logFC values with the corresponding colour code. 
biological process "C-type lectin receptors" were induced by ETEC infection (Figure 6). Two genes, AHCYL1 (adenosylhomocysteinase-like 1) and CALM3 (calmodulin 3), which are involved in the dectin-1-dependent calcineurin/NFAT pathway, were altered (AHCYL1, logFC of 2.57, and CALM3, logFC of -3.78). Activation of the AHCYL1 gene in all experimental groups was observed (Figure 6). AHCYL1 interacts with the inositol IP3 receptor and is involved in intracellular calcium release [33, 34]. Interestingly, the CALM3 gene was downregulated only in cells challenged with E. coli; hence, calmodulin could not activate calcineurin, and therefore, the transcription factor NFAT was not activated. This downregulation of the CALM3 gene was abolished by EPS pretreatment administered prior to ETEC infection.

Seven DEGs (NFKBIA, RELA, UBE2D3, UBE2V1, IKBKG, CHUK, and NFKB1) that participate in Dectin-1-mediated activation of NF- $\mathrm{KB}$ were found to be induced (Figure 6, Additional file 4). EPS treatment induced alterations in IKBKG, CHUK and NFKB1, while EPS pretreatment induced the upregulation of NFKB1 (Figure 6). The ETEC challenge induces the expression

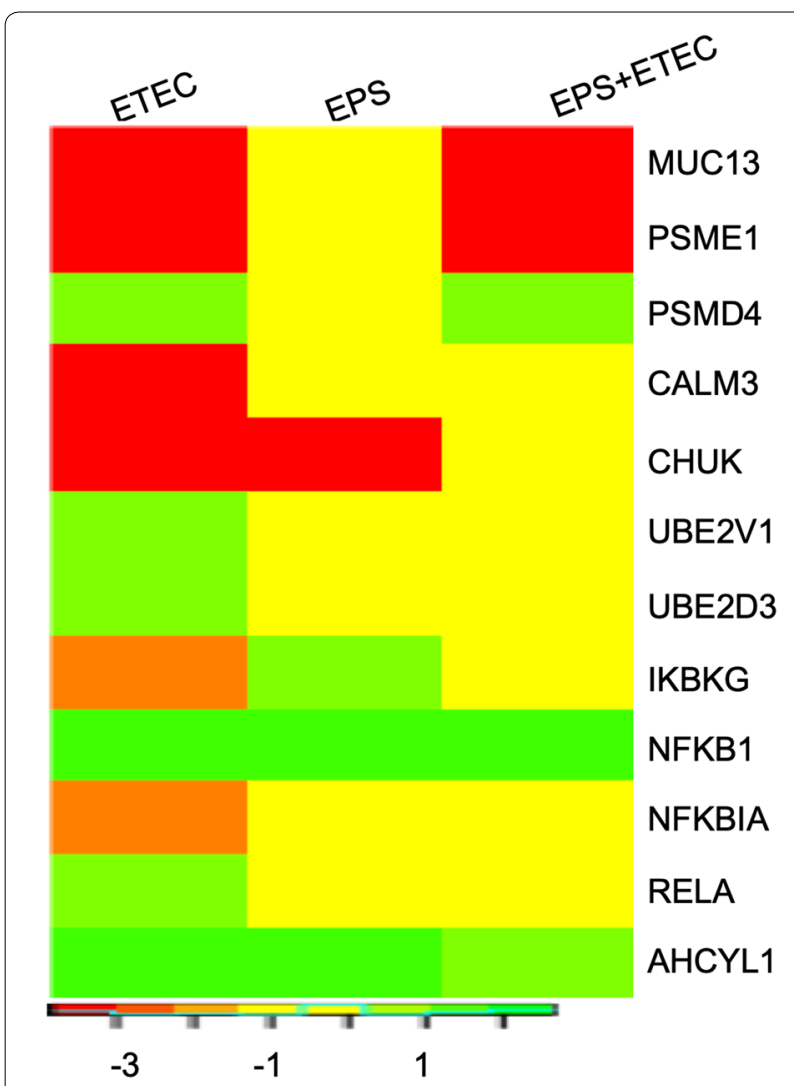

Figure 6 DEGs related to the GO biological process "CLR". Heat map showing the DEGs in the IPEC-1 cells challenged with ETEC, treated with EPS, or pretreated with EPS before ETEC challenge. The bar shows logFC values with the corresponding colour code. of the UBE2D3 and UBE2V1 genes (both with logFC of 1.48) encoding ubiquitin-conjugating enzyme E2. The UBE2D3 enzyme participates in the polyubiquitination of NF-kappa-B inhibitor alpha (IKBA), while the UBE2V1 enzyme forms a heterodimer complex with UBE2N (UBE2V1-UBE2N), which together with TRAF6, participates in the polyubiquitination of NF-kappa-B inhibitor gamma (IKBKG). Ubiquitination of inhibitors leads to the subsequent proteasomal degradation of the inhibitors; therefore, the upregulation of the UBE2D3 and $\mathrm{UBE} 2 \mathrm{~V} 1$ genes likely promotes the release of the NF- $\mathrm{kB}$ transcription factor [35]. The expression of these genes was unaltered in the EPS-treated cells and the pretreated cells challenged with ETEC. We also found downregulation of the NFKBIA gene encoding IKBA in the cells infected with ETEC ( $\operatorname{logFC}$ of -1.71$)$. This downregulation was abolished by EPS pretreatment prior to E. coli infection. The downregulation of NFKBIA was associated with the upregulation of the genes encoding two subunits of NF-kB: NFKB1, encoding the p105 subunit (logFC of 2.40), and RELA, encoding the p65 subunit (logFC of 2.13), in cells infected with E. coli (Additional file 5). The expression of the p105 subunit was also increased in cells treated with EPS $(\operatorname{logFC}$ of 2.42$)$ or pretreated before the E. coli challenge ( $\operatorname{logFC}$ of 2.65); however, in this case, the expression of the $\mathrm{p} 65$ subunit was unaffected.

\section{DEGs related to TLR cascades}

In the cells infected with E. coli, 20 induced DEGs (8 downregulated and 12 upregulated) $t$ are related to the GO biological process "Toll-like receptor cascades" (Figure 7). TLRs are major microbial pattern-recognition receptors. Eighteen DEGs (8 downregulated and 10 upregulated) were associated with the $\mathrm{GO}$ biological process "TLR4 cascade", and 14 DEGs (6 downregulated and 8 upregulated) were categorized in the "TLR2 cascade" process (Additional file 6). Although TLR4 was upregulated in each experimental group, the LBP gene encoding LPS-binding protein (LBP) was induced only in the ETEC-challenged cells ( $\operatorname{logFC}$ of 2.65). On the other hand, the expression of CD14- and Ly96 (also known as MD-2)-encoding genes was upregulated only after the ETEC challenge, irrespective of pretreatment with EPS.

The binding of a ligand to TLR4 initiates MyD88dependent and TRIF-dependent pathways [36]. Expression of the MyD88 gene was upregulated only in the case of ETEC challenge (with and without pretreatment, $\operatorname{logFC}$ of 2.54 and $\operatorname{logFC}$ of 2.75 , respectively). The activation of the MyD88-dependent pathway causes the activation of TAK1 (transforming growth factor (TGF)activating kinase 1); however, we did not observe altered expression of the genes participating in the activation of TAK1, except for the downregulation of IRAK-4 (which 


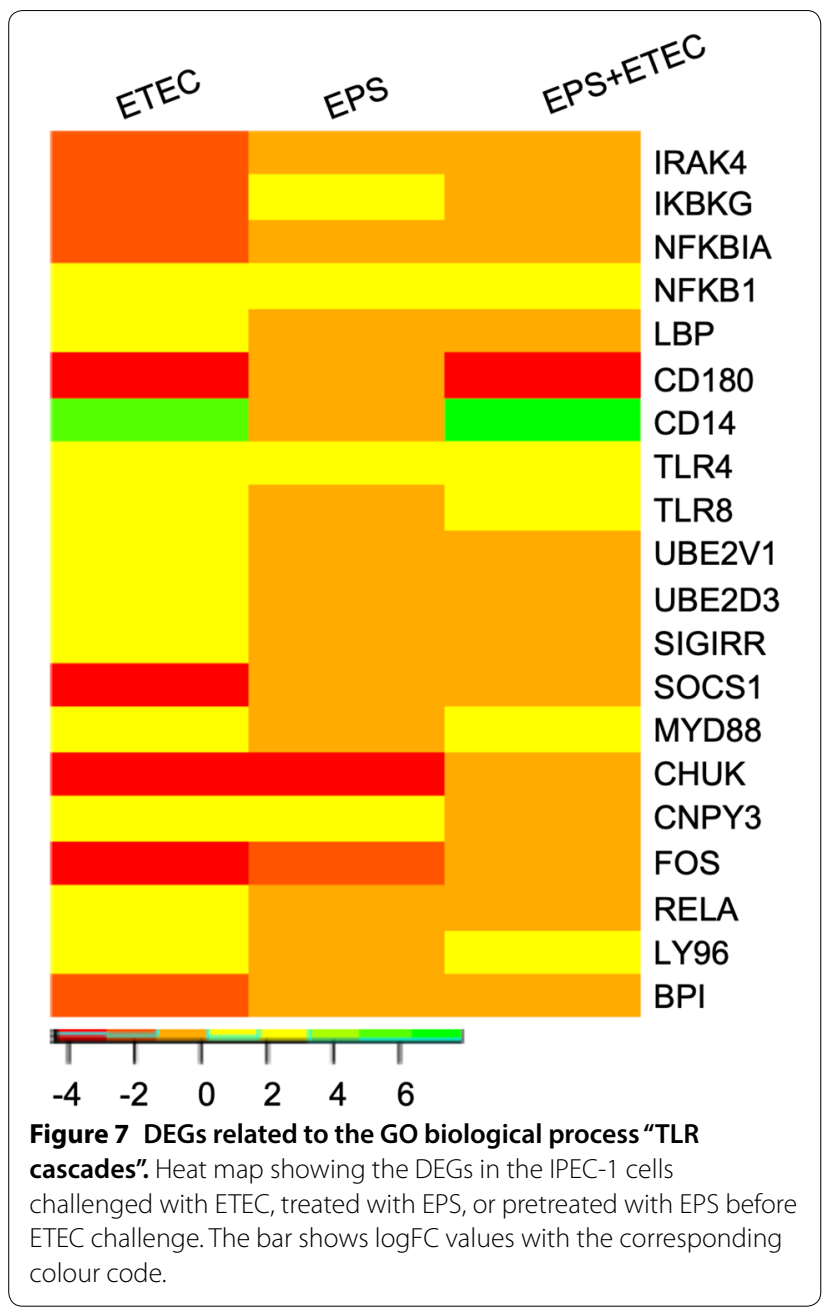

encodes IL-1R-associated kinase 4) in the ETEC-challenged cells ( $\log \mathrm{FC}$ of -1.77$)$. Activated TAK1 phosphorylates the IKB kinase (IKK) complex critical for the phosphorylation of the inhibitory protein I $\mathrm{K} B$ subunit of the NF- $\mathrm{kB}$ transcription factor. Three genes associated with the regulation of NF- $\mathrm{KB}$ were downregulated in the cells challenged with $E$. coli, namely, NFKBIA, encoding

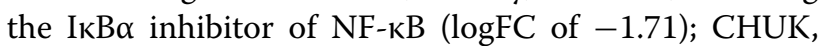
encoding the IKK $\alpha$ kinase subunit $(\operatorname{logFC}$ of -3.47$)$; and IKBKG, encoding the IKK $\gamma$ kinase subunit (logFC of $-1.79)$. Notably, the expression of these genes was unaltered in the cells pretreated with EPS and then challenged with $E$. coli (Figure 7). The ETEC challenge also induced the expression of the UBE2D3 and UBE2V1 genes (both with $\operatorname{logFC}$ of 1.48), which are involved in the GO biological process "MyD88-independent pathway". The expression of these genes was unaltered in the EPS-treated and pretreated cells challenged with ETEC.

Various negative regulatory mechanisms are necessary to attenuate TLR4 signalling and maintain immune system balance, including the activity of the TLR4-negative regulators CD180 (also known as RP105), SIGIRR (single Ig IL-1 receptor-related molecule), TNFAIP3 (TNF alpha-induced protein 3, also called A20) and SOCS1 (suppressor of cytokine signaling 1). The expression of CD180 was downregulated only in the case of ETEC challenge (with and without pretreatment, logFC of -4.26 and $\operatorname{logFC}$ of -3.73 , respectively), whereas treatment with only EPS had no effect on their expression levels. On the other hand, the expression of SIGIRR was induced in E. coli-infected cells (logFC of 2.09). The expression of TNFAIP3 was upregulated in the E. colichallenged cells (with and without pretreatment, $\operatorname{logFC}$ of 2.90 and $\operatorname{logFC}$ of 2.09 , respectively). The expression of SOCS1 was altered only in the ETEC-challenged cells ( $\log \mathrm{FC}$ of -4.38 ) (Figure 7 ).

\section{DEGs related to cytokine signalling}

Studying the ETEC-challenged cells, we observed 73 DEGs (17 downregulated and 56 upregulated) related to the GO biological process "Cytokine signaling in immune system" (Figure 8). As expected, E. coli infection of IPEC-1 cells induced the upregulation of genes encoding cytokines participating in the inflammatory response, such as IL1B2 (logFC of 3.07), TNF ( $\operatorname{logFC}$ of 2.01), IL6 ( $\operatorname{logFC}$ of 2.73$)$, IL2 ( $\operatorname{logFC}$ of 2.86$)$, IL4 $(\operatorname{logFC}$ of 1.46), IL12b ( $\operatorname{logFC}$ of 2.86) and IL23A ( $\operatorname{logFC}$ of 2.79) (Additional file 7). It is noteworthy that the expression of the TNF, IL12b, and IL4 genes was unaltered in the cells pretreated with EPS (Figure 8), while other the ILs were upregulated: IL1B2 (logFC of 4.94), TNF ( $\operatorname{logFC}$ of 2.59 ), IL4 ( $\operatorname{logFC}$ of 1.59$)$ and IL23A ( $\operatorname{logFC}$ of 3.03). In all the experimental groups, we observed the upregulation of macrophage colony-stimulating factor 1 (CSF1) and IL34, which play essential roles in the proliferation and differentiation of monocytes and macrophages. The upregulation of CSF1 in the EPS-treated cells found have an logFC value of 7.44. It is important to note that administration of the EPS treatment prior to E. coli infection attenuated CSF1 gene expression, $\operatorname{logFC}$ of 1.70 . On the other hand, no attenuating effect of the EPS pretreatment on IFNB1 (encoding interferon beta 1) was found. The logFC of this gene was 3.63 in the cells challenged with E. coli, while the $\operatorname{logFC}$ was 4.00 in the cells pretreated before infection.

The ETEC infection of IPEC-1 cells induced the downregulation of the CXCL2 gene (logFC of -3.59$)$, encoding a powerful neutrophil chemoattractant, and the IL10 gene $(\log \mathrm{FC}$ of -1.77$)$, encoding interleukin 10 , which has powerful anti-inflammatory functions (Figure 8). The expression of these genes was unaltered in the EPSpretreated cells challenged with ETEC, suggesting that EPS may enhance the pro-inflammatory response during 

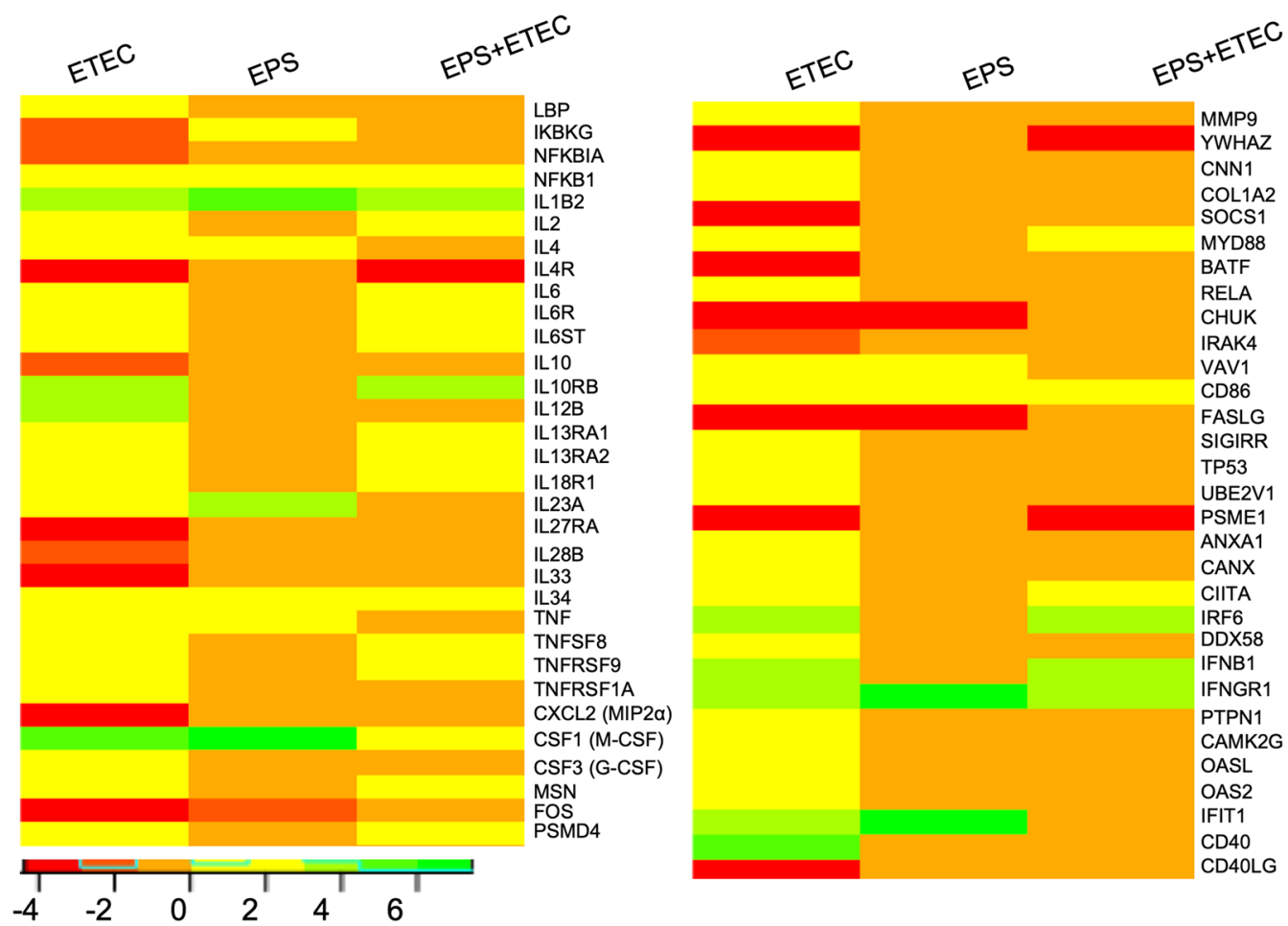

Figure 8 DEGs related to the GO biological process "Cytokine signaling in immune system". Heat map showing the DEGs in the IPEC-1 cells challenged with ETEC, treated with EPS, or pretreated with EPS before ETEC challenge. The bar shows logFC values with the corresponding colour code.

E. coli infection. The ETEC challenge of EPS-treated and untreated cells also induced upregulation of genes encoding receptors for some cytokines, such as IL6R, IL10RB, IL13RA, IL18R1, TNFRSF1A and TNFRSF9. The expression of these genes was unaltered in the EPS-treated and pretreated cells challenged with ETEC (Figure 8).

The $E$. coli infection of IPEC-1 cells induced the upregulation of the IFNB1 gene encoding interferon beta 1 ( $\operatorname{logFC}$ of 3.63$)$ and the IFNGR1 gene $(\operatorname{logFC}$ of 3.55$)$, encoding the ligand-binding alpha chain of the gamma interferon receptor. Expression of the IFNB1 gene was upregulated in the EPS-pretreated cells challenged with ETEC ( $\operatorname{logFC} 4.00)$ but was unaltered in the EPS-treated cells. The IFNGR1 gene was upregulated in all experimental groups, with expression level highest in the EPStreated cells ( $\operatorname{logFC}$ of 6.84) (Figure 8).

\section{Discussion}

ETECs adhere to epithelial cells through the interaction of fimbrial and non-fimbrial adhesins with cell surface receptors. Upon attachment, they release LT and/or ST enterotoxins, which act upon intestinal enterocytes by disrupting electrolyte homeostasis, resulting in fluid loss and eventually secretory diarrhea [37, 38]. LTs activate adenylate cyclase, which substantially increases the intracellular concentration of cAMP [39]. The increased concentration of cAMP leads to the activation of cAMPdependent kinase protein, which then stimulates chloride channels and inhibits $\mathrm{Na}^{+}$uptake [9]. In the current study, in E. coli-infected cells, the expression of adenylate cyclase-activating polypeptide (ADCYAP1 gene) was downregulated, while the ADCYAP1R1 gene encoding the receptor for ADCYAP1 was upregulated in all experimental groups (Additional file 8: Data set 1.7). Notably, some of the cAMP-dependent kinase proteins (PRKAG3 and PRKAR1A) were upregulated in the E. coli-infected cells, while EPS pretreatment before infection abolished the induction of PRKAR1A (Additional file 8: Data set 1.5). STb, one of the two classes of heat stable toxins [9, 30], stimulates a GTP-binding regulatory protein, resulting in an increase in intracellular $\mathrm{Ca}^{2+}$ levels activating $\mathrm{Ca}^{2+} /$ calmodulin-dependent protein kinase II (CAMKII), which opens a calcium-activated chloride channel. E. coli induced the regulation of CAMKII (CAMK2G); however, the upregulation was attenuated by EPS pretreatment (Figure 8). Thus, EPS pretreatment can reduce the overexpression of PRKAR1A and CAMK2G, which are activated in $E$. coli infection. Considering the crucial role of PRKAR1A and CAMK2G in electrolyte loss (mediated by the activation of ion channels), it is tempting to speculate 
that suppression of the induction of both genes by the EPS of $L$. reuteri prevents the development of diarrhea in ETEC-infected piglets. A similar effect was reported in a recent report, wherein authors demonstrated that pretreatment of IPEC-J2 cells with various strains of $L$. reuteri reduced the detrimental effect of $E$. coli enterotoxins on the mucosal barrier [40]. ETECs can disturb the TJs between epithelial cells $[9,41,42]$. Claudins are the backbone of TJs and can modulate the paracellular route of transport [43]. Nassour and Dubreuil demonstrated that the elevated intracellular $\mathrm{Ca}^{2+}$ levels in response to $\mathrm{STb}$ enterotoxins redistribute claudin- 1 from the plasma membrane to the cytosol, leading to an increase in paracellular permeability [44]. Similarly, in the current study, we found upregulation of the CLDN2 and CLDN2D genes in E. coli-challenged cells, while the expression of these genes was unchanged in the EPS-treated or pretreated cells. We also observed the upregulation of the PARD6B gene in the nontreated as well as pretreated cells infected with ETEC (Figure 3). This gene (a member of the PAR6 family) encodes a protein involved in the cell polarization process [45]. JAMs are among other induced molecules that participate in the generation of the cell junctional system and TJ assembly [41]. Similarly, the F11R gene, encoding the receptor for JAM-1, was also upregulated in the ETEC-challenged cells, but its expression was unchanged in the other two groups (Figure 3). Based on our results, we deduced that EPS pretreatment can reduce the overexpression of the genes participating in the formation of TJs caused by ETEC infection.

The ECM is a highly dynamic structure constantly undergoing a remodeling process [46]. The most significant enzymes participating in remodulation are metalloproteinases, which may degrade ECM. The metalloproteinases are categorized into matrix metalloproteinases (MMPs) and the ADAM (a disintegrin and metalloprotease domain) family [47]. In the current study, the ADAM17, ADAM10, MMP14 and MMP9 genes were induced in the ETEC-challenged cells (Figure 4), whereas, except for MMP14, their expression in the EPS-treated and pretreated cells remained unchanged. Thus, it can be assumed that the EPS of $L$. reuteri could reduce the ECM degradation induced by $E$. coli infection. We also observed that the ELN gene, encoding elastin, was downregulated in the ETECinfected cells, while in the EPS-treated or pretreated cells, it remained unchanged. A similar effect of EPS pretreatment was observed for the set of genes (ITGB3 and ITGA2B) encoding integrins (Figure 4). Integrins act as receptors mainly for extracellular matrix proteins [48], which mediate downstream gene transcription through a variety of signalling pathways, for example, via NF- $\mathrm{kB}$. In the present study, the activation of
NF- $\mathrm{KB}$ was noticed in E. coli-infected cells (Additional file 5). To our knowledge, no work has been published to date that explains the altered expression of integrins in IPEC-1 cells upon ETEC infection.

TLR signalling pathways play crucial roles in the regulation and activation of numerous pro-inflammatory molecules. LPS is a well-known activator of TLR4 [36]; however, recent studies have shown that EPS from lactic acid bacteria can also activate TLR4, TLR2 [5] and C-type lectin signalling pathways [7]. Activation of TLR4 induced downstream MyD88-dependent and TRIFdependent (MyD88-independent) pathways, which in turn led to a cascade of activated molecules, such as NF- $\mathrm{KB}$, activator protein-1 (AP-1), interferon regulatory factor 5 (IRF5) and IRF3 (type I interferons) and, finally, inflammatory cytokines [49] (Additional file 6). We have previously demonstrated that ETEC infection of IPEC-1 cells induces the expression of the NF-kB, IL1 $\beta$, TNF $\alpha$, and IL6 genes; however, EPS-L26 pretreatment before ETEC infection abates the overexpression of NF- $\mathrm{kB}$ and IL1 $\beta$ but not that of the TNF $\alpha$ or IL6 genes [6]. Such anti-inflammatory properties of EPS have also been demonstrated by others $[5,50,51]$. In the current work, we presumed that the NF- $\mathrm{kB}$ pathway was activated in the cells infected with $E$. coli because increased expression of IL2, IL4, IL6, IL12B and TNFa was found (Figure 8). A plausible anti-inflammatory effect of EPS was observed in this study, as the expression of the IL12B, TNF, CSF1 and CSF3 genes was abated in the cells pretreated before E. coli infection (Figure 8).

In conclusion, the transcriptomic analyses performed in this study showed differential cellular responses to ETEC infection with or without pretreatment with the EPS derived from $L$. reuteri. This study allowed us to obtain a global perspective on the induced genes and pathways involved in the cellular response to EPS and $E$. coli alone and in combination. The results indicate that the use of EPS may be a good strategy to improve intestinal homeostasis. EPS modulated the genes participating in the formation of TJs. EPS also attenuated the overexpression of genes known to be activated by LT or ST, which are associated with electrolyte loss. Furthermore, the attenuation of the expression of the genes encoding proteases was observed in the cells pretreated with EPS pretreated compared to those not pretreated before $E$. coli infection. The most relevant finding of this study is that EPS has a suppressive effect on the inflammatory response evoked by E. coli infection. Overall, the data map offers a comprehensive picture of the positive effect of EPS pretreatment and provides sound benchmarking for further studies, which should be conducted using other omics approaches to validate the positive effects of EPS. 


\section{Supplementary information}

Supplementary information accompanies this paper at https://doi. org/10.1186/s13567-020-00773-1.

Additional file 1. Analysis of the genes expressed in the IPEC-1 cells challenged with ETEC, treated with EPS, or pretreated with EPS before ETEC challenge by using Reactome. IPEC-1 cell +ETEC: analysis based on the gene names of 463 entities; IPEC-1 cells + EPS: analysis based on the gene names of 111 entities; and IPEC-1 cells + EPS + ETEC: analysis based on the gene names of 147 entities.

Additional file 2. DEGs involved in the tight junction organization pathway. DEGs found in the study are highlighted in the pathway "tight junction organization" retrieved from the KEGG database. Red indicates the genes expressed in the cells challenged with ETEC. Blue indicates genes expressed in the cells treated with EPS (note, no genes in this pathway were induced by this treatment). Green indicates genes expressed in the cells pretreated with EPS and challenged with ETEC.

Additional file 3. DEGs involved in the ECM-receptor interaction pathway. DEGs involved in the ECP-receptor interaction pathway (retrieved from the KEGG database) as highlighted in three experimental groups. Red indicates genes expressed in the cells challenged with ETEC. Blue indicates genes expressed in the cells treated with EPS. Green indicates genes expressed in the cells pretreated with EPS and challenged with ETEC.

Additional file 4. DEGs involved in the CLR signalling pathway. The KEGG results showing the C-type lectin receptor signalling pathway. DEGs were highlighted on the basis of three experimental groups. Red indicates genes expressed in the cells challenged with ETEC. Blue indicates genes expressed in the cells treated with EPS. Green indicates genes expressed in the cells pretreated with EPS and challenged with ETEC.

Additional file 5. DEGs involved in the NF kappa B signalling pathway. DEGs involved in the NF kappa B signalling pathway are highlighted on the basis of three experimental groups. Red indicates genes expressed in the cells challenged with ETEC. Blue indicates genes expressed in the cells treated with EPS. Green indicates genes expressed in the cells pretreated with EPS and challenged with ETEC.

\section{Additional file 6. DEGs involved in the TLR signalling pathway.}

DEGs in the TLR signalling pathway found in three cell treatments are highlighted. Red indicates genes expressed in the cells challenged with ETEC. Blue indicates genes expressed in the cells treated with EPS. Green indicates the genes expressed in cells pretreated with EPS and challenged with ETEC.

Additional file 7. DEGs involved in the cytokine-cytokine receptor interaction pathway. DEGs from the cytokine-cytokine receptor interaction pathway found in the cells subjected to one of the three treatments are highlighted. Red indicates genes expressed in the cells challenged with ETEC. Blue indicates genes expressed in the cells treated with EPS Green indicates genes expressed in the cells pretreated with EPS and challenged with ETEC.

Additional file 8. Genes identified through RNA-seq in the downstream bioinformatics analysis. Data sets from 1.1 to 1.3-Differentially expressed genes (DEGs) found in the cells subjected to one of the three treatments. Data sets from 1.4 to 1.7-Common genes identified among the treated cells. Data sets from 1.8 to 1.10—Molecular pathways identified in the treated cells.

\section{Acknowledgements}

This work was supported by VEGA 1/0633/17 (100\%). PT is supported by H2O20-MSCA-ITN-2017-EJD: Marie Skłodowska-Curie Innovative Training Networks 765423. EM is supported by APVV-18-0259. We acknowledge the CF Genomics CEITEC MU, supported by the NCMG research infrastructure (LM2015091 funded by MEYS CR), for its support in obtaining the NGS data.

\section{Authors' contributions}

LT conceived the project. The cell culture was established by EM. The cell challenge was performed by EK and LT. RNA isolation and library preparation were completed by EK. The bioinformatics analysis was performed by TP and MB. TP, ZK and MB prepared the manuscript. All authors read and approved the final manuscript.

\section{Funding}

This work was supported by VEGA 1/0633/17 (100\%). PT is supported by H2020-MSCA-ITN-2017-EJD: Marie Skłodowska-Curie Innovative Training Networks 765423. EM is supported by APVV-18-0259.

\section{Competing interests}

The authors declare that they have no competing interests.

\section{Author details}

${ }^{1}$ Institute of Immunology, University of Veterinary Medicine and Pharmacy in Kosice, Kosice, Slovakia. ${ }^{2}$ Laboratory of Biomedical Microbiology and Immunology, University of Veterinary Medicine and Pharmacy in Kosice, Kosice, Slovakia. ${ }^{3}$ Institute of Neuroimmunology, SAV, Dubravska cesta 9, Bratislava, Slovakia.

Received: 9 December 2019 Accepted: 5 February 2020

Published online: 31 March 2020

\section{References}

1. Chapot-Chartier MP, Kulakauskas S (2014) Cell wall structure and function in lactic acid bacteria. Microb Cell Fact 13:59

2. Badel S, Bernardi T, Michaud P (2011) New perspectives for Lactobacilli exopolysaccharides. Biotechnol Adv 29:54-66

3. Oleksy M, Klewicka E (2018) Exopolysaccharides produced by Lactobacillus sp.: biosynthesis and applications. Crit Rev Food Sci Nutr 58:450-462

4. Bleau C, Monges A, Rashidan K, Laverdure JP, Lacroix M, Van Calsteren MR, Millette M, Savard R, Lamontagne L (2010) Intermediate chains of exopolysaccharides from Lactobacillus rhamnosus RW-9595M increase IL-10 production by macrophages. J Appl Microbiol 108:666-675

5. Laiño J, Villena J, Kanmani P, Kitazawa H (2016) Immunoregulatory effects triggered by lactic acid bacteria exopolysaccharides: new insights into molecular interactions with host cells. Microorganisms 4:E27

6. Kšonžeková P, Bystrický P, Vlčková S, Pätoprstý V, Pulzová L, Mudroňová D, Kubašková T, CsankT, Tkáčiková L (2016) Exopolysaccharides of Lactobacillus reuteri: their influence on adherence of E. coli to epithelial cells and inflammatory response. Carbohydr Polym 141:10-19

7. Wells JM, Rossi O, Meijerink M, van Baarlen P (2011) Epithelial crosstalk at the microbiota-mucosal interface. Proc Natl Acad Sci USA 108:4607-4614

8. Rescigno M (2010) Intestinal dendritic cells. Adv Immunol 107:109-138

9. Dubreuil JD, Schifferli DM (2016) Animal enterotoxigenic Escherichia coli. EcoSal Plus 7: ESP-0006-2016

10. Nagy B, Fekete PZ (1999) Enterotoxigenic Escherichia coli (ETEC) in farm animals. Vet Res 30:259-284

11. Zhou C, Liu Z, Jiang J, Yu Y, Zhang Q (2012) Differential gene expression profiling of porcine epithelial cells infected with three enterotoxigenic Escherichia coli strains. BMC Genomics 13:330

12. Devriendt B, Verdonck F, Summerfield A, Goddeeris BM, Cox E (2010) Targeting of Escherichia coli F4 fimbriae to Fcy receptors enhances the maturation of porcine dendritic cells. Vet Immunol Immunopathol 135:188-198

13. Kobayashi H, Albarracin L, Sato N, Kanmani P, Kober AKMH, Ikeda-Ohtsubo W, Suda Y, Nochi T, Aso H, Makino S, Kano H, Ohkawara S, Saito T, Villena J, Kitazawa H (2016) Modulation of porcine intestinal epitheliocytes immunetranscriptome response by Lactobacillus jensenii TL2937. Benef Microbes 7:769-782

14. Albarracin L, Kobayashi H, lida H, Sato N, Nochi T, Aso H, Salva S, Alvarez S, Kitazawa H, Villena J (2017) Transcriptomic analysis of the innate antiviral immune response in porcine intestinal epithelial cells: influence of immunobiotic lactobacilli. Front Immunol 8:57

15. Taranu I, Marin DE, Braicu C, Pistol GC, Sorescu I, Pruteanu LL, Neagoe IB, Vodnar DC (2018) In vitro transcriptome response to a mixture of lactobacilli strains in intestinal porcine epithelial cell line. Int J Mol Sci 19:E1923 
16. Hulst M, Van Der Weide R, Hoekman A, Van Krimpen M (2019) Transcriptional response of cultured porcine intestinal epithelial cells to micro algae extracts in the presence and absence of enterotoxigenic Escherichia coli. Genes Nutr 14:8

17. Releases, alexdobin/STAR, GitHub. https://github.com/alexdobin/STAR/ releases. Accessed 18 Feb 2020

18. Bioconductor-Open Source Software For Bioinformatics. https://bioco nductor.org/packages/release/bioc/html/edgeR.html. Accessed 18 Feb 2020

19. Base 2 Logarithm, Log2 Calculator-EndMemo. http://www.endme mo.com/algebra/log2.php. Accessed 18 Feb 2020

20. Pearson Correlation Coefficient Calculator. https://www.socscistatistic s.com/tests/pearson/default2.aspx. Accessed 18 Feb 2020

21. ELIXIR (2018) Reactome Pathway Database. In: reactome. https://react ome.org/. Accessed 18 Feb 2020

22. Expression Heat Map. http://www.heatmapper.ca/expression/. Accessed 18 Feb 2020

23. KEGG: Kyoto Encyclopedia of Genes and Genomes. https://www.genom e.jp/kegg/. Accessed 18 Feb 2020

24. Hartsock A, Nelson WJ (2008) Adherens and tight junctions: structure, function and connections to the actin cytoskeleton. Biochim Biophys Acta 1778:660-669

25. Anderson JM, Van Itallie CM (2009) Physiology and function of the tight junction. Cold Spring Harb Perspect Biol 1:a002584

26. Walko G, Castañón MJ, Wiche G (2015) Molecular architecture and function of the hemidesmosome. Cell Tissue Res 360:363-378

27. Alarcon VB (2010) Cell polarity regulator PARD6B is essential for trophectoderm formation in the preimplantation mouse embryo. Biol Reprod 83:347-358

28. Markmann A, Hausser H, Schönherr E, Kresse H (2000) Influence of decorin expression on transforming growth factor- $\beta$-mediated collagen gel retraction and biglycan induction. Matrix Biol 19:631-636

29. Imai K, Hiramatsu A, Fukushima D, Pierschbacher MD, Okada Y (1997) Degradation of decorin by matrix metalloproteinases: identification of the cleavage sites, kinetic analyses and transforming growth factor- $\beta 1$ release. Biochem J 322:809-814

30. Danen EHJ, Sonnenberg A (2003) Integrins in regulation of tissue development and function. J Pathol 200:471-480

31. Hatori Y, Lutsenko S (2016) The role of copper chaperone Atox 1 in coupling redox homeostasis to intracellular copper distribution. Antioxidants (Basel) 5:E25

32. Park BS, Lee JO (2013) Recognition of lipopolysaccharide pattern by TLR4 complexes. Exp Mol Med 45:e66

33. Ando H, Mizutani A, Tsuzurugi D, Michikawa T (2006) IRBIT suppresses IP3 receptor activity by competing with IP3 for the common binding site on the IP3 receptor. Mol Cell 22:795-806

34. Ando H, Kawaai K, Mikoshiba K (2014) IRBIT: a regulator of ion channels and ion transporters. Biochim Biophys Acta 1843:2195-2204

35. Collins PE, Mitxitorena I, Carmody RJ (2016) The ubiquitination of NF-KB subunits in the control of transcription. Cells 5:E23
36. Takeda K, Akira S (2004) TLR signaling pathways. Semin Immunol 16:3-9

37. Wang H, Zhong Z, Luo Y, Cox E, Devriendt B (2019) Heat-stable enterotoxins of enterotoxigenic Escherichia coli and their impact on host immunity. Toxins 11:E24

38. Yi H, Wang L, Xiong Y, Wang Z, Qiu Y, Wen X, Jiang Z, Yang X, Ma X (2018) Lactobacillus reuteri LR1 improved expression of genes of tight junction proteins via the MLCK pathway in IPEC-1 cells during infection with enterotoxigenic Escherichia coli K88. Mediators Inflamm 2018:6434910

39. Hajishengallis G, Connell TD (2013) Type II heat-labile enterotoxins: structure, function, and immunomodulatory properties. Vet Immunol Immunopathol 152:68-77

40. Karimi S, Jonsson H, Lundh T, Roos S (2018) Lactobacillus reuteri strains protect epithelial barrier integrity of IPEC-J2 monolayers from the detrimental effect of enterotoxigenic Escherichia coli. Physiol Rep 6:e13514

41. Dubreuil JD (2017) Enterotoxigenic Escherichia coli targeting intestinal epithelial tight junctions: an effective way to alter the barrier integrity. Microb Pathog 113:129-134

42. Guttman JA, Finlay BB (2009) Tight junctions as targets of infectious agents. Biochim Biophys Acta 1788:832-841

43. Krause G, Winkler L, Mueller SL, Haseloff RF, Piontek J, Blasig IE (2008) Structure and function of claudins. Biochim Biophys Acta 1778:631-645

44. Nassour H, Dubreuil JD (2014) Escherichia coli STb enterotoxin dislodges claudin-1 from epithelial tight junctions. PLoS One 9:e113273

45. Zhou Q, Dai J, Chen T, Dada LA, Zhang X, Zhang W, DeCamp MM, Winn RA, Sznajder JI, Zhou G (2017) Downregulation of PKCZ/Pard3/Pard6b is responsible for lung adenocarcinoma cell EMT and invasion. Cell Signal 38:49-59

46. Lu P, Takai K, Weaver VM, Werb Z (2011) Extracellular matrix degradation and remodeling in development and disease. Cold Spring Harb Perspect Biol 3:a005058

47. Cawston TE, Young DA (2010) Proteinases involved in matrix turnover during cartilage and bone breakdown. Cell Tissue Res 339:221-235

48. Gilcrease MZ (2007) Integrin signaling in epithelial cells. Cancer Lett 247:1-25

49. Zughaier SM, Zimmer SM, Datta A, Carlson RW, Stephens DS (2005) Differential induction of the toll-like receptor 4-MyD88-dependent and -independent signaling pathways by endotoxins. Infect Immun 73:2940-2950

50. Du B, Yang Y, Bian Z, Xu B (2017) Characterization and anti-inflammatory potential of an exopolysaccharide from submerged mycelial culture of Schizophyllum commune. Front Pharmacol 8:252

51. Chen Y, Zhang M, Ren F (2019) A role of exopolysaccharide produced by Streptococcus thermophilus in the intestinal inflammation and mucosal barrier in caco-2 monolayer and dextran sulphate sodium-induced experimental murine colitis. Molecules 24:E513

\section{Publisher's Note}

Springer Nature remains neutral with regard to jurisdictional claims in published maps and institutional affiliations.

Ready to submit your research? Choose BMC and benefit from

- fast, convenient online submission

- thorough peer review by experienced researchers in your field

- rapid publication on acceptance

- support for research data, including large and complex data types

- gold Open Access which fosters wider collaboration and increased citations

- maximum visibility for your research: over $100 \mathrm{M}$ website views per year

At BMC, research is always in progress.

Learn more biomedcentral.com/submissions 